\title{
Cancer Screening Adherence of Asian Women According to Biochemically-verified Smoking Status: Korea National Health and Nutrition Examination Survey
}

\author{
Young-Jin Ko', Soyeun Kim¹, Kyae-Hyung Kim², Kiheon Lee ${ }^{3}$, Cheol Min \\ Lee $^{4 *}$
}

\section{Abstract}

Background: Men and women who smoke tend to show less compliance to screening guidelines than nonsmokers. However, a recent study in Korea showed that self-reported female smokers constituted less than half of cotinine-verified smokers. Therefore, the aim of this study was to identify hidden smokers using cotinineverified method and examine cancer screening behavior according to biochemically verified smoking status. Materials and Methods: Among 5,584 women aged 30 years and older who participated in the Fourth and Fifth Korea National Health and Nutrition Examination Survey (KNHANES), 372 (6.66\%) hidden smokers were identified based on interview responses and verified by urinary cotinine levels. We compared cancer-screening behavior (cervical, breast, stomach, and colon cancer) of female hidden smokers to that of non-smokers and selfreported smokers by cross-sectional analysis. Results: Hidden female smokers had significantly lower adherence to breast cancer screening compared to non-smokers (aOR (adjusted odds ratio) $[95 \% \mathrm{CI}]=0.71[0.51-0.98]$ ). Adherence to stomach cancer $(\mathrm{aOR}[95 \% \mathrm{CI}]=0.75[0.54-1.03])$ and cervical cancer $(\mathrm{aOR}[95 \% \mathrm{CI}]=\mathbf{0 . 8 5}$ [0.66-1.10]) screening was also lower among hidden female smokers compared to non-smokers. Self-reported (current) smokers showed lowest adherence to cervical cancer (aOR: 0.64, 95\% CI0.47-0.87), breast cancer $(0.47$ [0.32-0.68]), stomach cancer $(0.66[0.46-0.95])$, and colon cancer $(0.62$ [0.38-1.01]) screening compared to non-smokers, followed by female hidden smokers, then non-smokers. These lower adherence rates of current smokers were attenuated after we incorporated hidden smokers into the current smoker group. Conclusions: Cancer screening adherence of female hidden smokers was lower than cotinine-verified non-smokers but higher than current smokers. Considering the risk of smoking-related cancer among women, identifying hidden smokers is important to encourage appropriate cancer screening.

Keywords: Cancer screening - hidden smoker - female smoker

Asian Pac J Cancer Prev, 16 (9), 4081-4088

\section{Introduction}

Cigarette smoking is the leading preventable cause of death (Center for Disease Control and Prevention, 2008; World Health Organization, 2011) and is known to cause cancer of various organs (Vineis et al., 2004). Smoking is especially associated with increased incidence of colon cancer (Botteri et al., 2008), breast cancer (Johnson et al., 2011; Gaudet et al., 2013), stomach cancer (Ladeiras-Lopes et al., 2008), and uterine/cervical cancer (International collaboration of epidemiological studies of cervical cancer, 2006; International collaboration of epidemiological studies of cervical cancer, 2007). Screening for breast, stomach, cervical, and colon cancer are highly recommended in the national screening program in Korea.
Smoking is prejudicial to the health of smokers. Furthermore, smokers tend to have other unhealthy health behavior (Berrigan et al., 2003; Ruidavets et al., 2004; de Vries et al., 2008). Previous studies reported that smokers show less compliance to screening guidelines than non-smokers (Shapiro et al., 2001; Carlos et al., 2005; Rakowski et al., 2005; Rakowski et al., 2006).

In general, defining smokers based on self-reporting is regarded as reliable in population-based surveys in Western populations (West et al., 2007; Yeager and Krosnick, 2010; Wong et al., 2012). However, in East Asian countries with very low reported smoking rates in women, under-reporting of hidden female smokers is emerging as an issue. In fact, several studies reported an underestimation of the true number of smokers in Asian populations (Jung-Choi et al., 2012; Wang and Ma, 2012).

${ }^{1}$ Department of Family Medicine, Korea Cancer Center Hospital, ${ }^{2}$ Department of Family Medicine, Seoul National University Hospital, ${ }^{4}$ Department of Family Medicine, Healthcare System Gangnam Center, Seoul National University Hospital, Seoul, ${ }^{3}$ Department of Family Medicine, Seoul National University Bundang Hospital, Seoul National University College of Medicine, Kyoung-gi, Korea *For correspondence: bigbangx.snuh@gmail.com 
A recent study in Korea showed that self-reported female smokers $(5.3 \%)$ constituted only half of cotinine-verified smokers (13.9\%), using national representative data (JungChoi et al., 2012). In contrast, the ratio of cotinine-verified to self-reported smoking rates was 1.12 for men. This gender difference in under-reporting of smoking warrants further attention into the consequences of hidden female smokers. However, to the best of our knowledge, health behavior, especially in terms of cancer screening in hidden female smokers, has not yet been investigated.

Therefore, in this study, we identified hidden smokers among reported non-smokers using the cotinineverification method and then examined cancer-screening behavior in hidden smokers, non-smokers, self-reported (current) smokers, and former smokers.

\section{Materials and Methods}

\section{Study subjects}

Data were derived from the 2008, 2009, and 2010 Korea National Health and Nutrition Examination Survey (KNHANES). The KNHANES is a series of national health surveys in Korea that use a stratified multistage probability sampling design to select a representative sampling of the Korean population. The KNHANES "IV and $\mathrm{V}$ health interview surveys were conducted through face-to-face interviews at the homes of subjects by trained interviewers. Informed consent was given by each participant prior to inclusion in the study (Korea Centers for Disease Control \& Prevention, 2007). We used data from the KNHANES IV (2008-2009) and V (2010), which contain information on urine cotinine level, to identify hidden smokers.

Initial candidates for this study were 15,907 women who completed both a health examination survey and a health interview survey. Of these, women less than 30 years of age $(n=5,231)$ were excluded. Of the remaining women, participants whose urinary cotinine was not measured ( $n=4,977)$, those who did not respond to the smoking interview $(\mathrm{n}=17)$, and those with missing information on education, marital status, body mass index (BMI), or alcohol consumption or those who did not respond all questions of the cancer screening interview $(n=94)$ were excluded. Therefore, a total of 5,584 participants were included in the study (Figure 1).

Cervical cancer screening is recommended for women over 30 years of age, breast cancer and stomach cancer screening is recommended for women over 40 years of age, and colon cancer screening is recommend after 50 years. Therefore, the numbers of patients in each screening population for different cancer types differs, as per the recommended guidelines.

\section{Smoking measure}

All respondents were asked if they had smoked more than a total of 100 cigarettes in their life and if they were current smokers . Respondents who reported having consumed $<100$ cigarettes in their lifetime and not being current smokers were considered "non-smokers". Those who consumed more than 100 cigarettes in their lifetime were divided into those who currently smoked "self-reported (current) smokers" and those who did not currently smoke "former smokers". Among the nonsmokers and former smokers, those whose urinary cotinine concentrations higher than $50 \mathrm{ng} / \mathrm{mL}$ were defined as cotinine-verified hidden smokers (Haufroid and Lison, 1998; SNRT Subcommittee on Biochemical Verification, 2002).

Urinary cotinine levels were measured by tandem mass spectrometry with Tandem mass API 4000 (Applied Biosystems, Carisbad, CA,USA) and by gas chromatography and mass spectrometry with Perkin Elmer Clarus 600T (PerkinElmer, Turku, Finland) (Korea Centers for Disease Control \& Prevention, 2010), which has a threshold of detection of $0.25 \mathrm{ng} / \mathrm{mL}$.

\section{Cancer screening adherences}

Using the KHANES for each cancer type, patients were asked whether they had ever had a screening test, and if so, the length of time since the last test.

The following dependent variables and populations were used in the analysis. The National Cancer Screening Program was used to determine compliance (Jun-Sik, 2010). Stomach cancer screening: esophagogastroduodenoscopy or upper GI series within 2 years in patients older than 40 years. Breast cancer screening: mammography or breast ultrasonography within 2 years in patients older than 40 years. Cervical cancer screening: Pap smear test within 2 years in patients older than 30 years. Colon cancer screening: occult blood test within 2 years, colonoscopy within 10 years, or sigmoidoscopy and double contrast study within 10 years in patients older than 50 years.

\section{Independent variables}

We collected data on independent variables from KNHANES IV and V. Variables included sociodemographic factors (age, BMI, education level, marital status, insurance status, and private insurance), behavioral risk factors (alcohol consumption and exercise), and personal health status (history of chronic disease such as hypertension, diabetes, and dyslipidemia and history of cancer).

Socio-demographic variables were current age (30-39, $40-49,50-64$, or 65 years and over), education (less than elementary, middle/high, or college or higher), marital status (living with spouse or without spouse), national health insurance (NHI (national health insurance), Medicaid, or not involved in either), private health insurance (no, yes, or do not know), residential area (urban or rural) and obesity (BMI $<25 \mathrm{~kg} / \mathrm{m}^{2}$ or BMI $\geq 25 \mathrm{~kg} / \mathrm{m}^{2}$ ) (World Health Organization, 2000).

Behavioral risk factors were physical activity of moderate intensity (yes or no) and alcohol consumption (non-drinker, less than 1 drink per month, or more than 1 drink per month). Physical activity of moderate intensity was defined as engaging in vigorous activity of at least 20 minutes per day for 3 or more days, moderate-intensity activity or walking of at least 30 minutes per day for 5 or more days, or any combination of walking, moderateintensity, or vigorous intensity activities achieving a minimum of at least $600 \mathrm{MET}-\mathrm{min} /$ week using IPAQ 
(International Physical Activity Questionnaires) shortform questionnaire for 5 or more days (The IPAQ group, 2005). Personal health status was assessed as: diagnosis of hypertension, diabetes, and dyslipidemia, and history of cancer by doctor.

\section{Statistical methods}

Participants were categorized into four groups according to their smoking status, as follows: non-smoker, hidden smoker, self-reported (current) smoker, and former smoker.

Baseline characteristics are expressed as absolute number $(\%)$ according to the smoking status. $\chi^{2}$ tests and ANOVA were used to compare categorical variables and continuous variables, respectively, according to smoking status. Levels of urinary cotinine were expressed as median (interquartile range) because of their skewed distribution.

We used a weighted population sample to reflect the sampling method and response rate. We calculated the estimated proportions and standard errors for the subgroups based on each variable.

We calculated the odds ratio (OR) and $95 \%$ confidential interval $(\mathrm{CI})$ to determine the association between smoking status and cancer screening. After adjusting for potential confounders in gradual modeling, a multiple logistic regression method was used. In model 1, we adjusted for socio-demographic factors such as age, BMI, education level, marital status, insurance status, and private insurance. In model 2 , we also adjusted for socio-demographic factors and behavioral risk factors (alcohol consumption and exercise). In model 3, we also adjusted for socio-demographic factors, behavioral risk factors, and personal health status (history of chronic disease (e.g. hypertension, diabetes, and dyslipidemia) and history of cancer).

We also investigated cancer screening and smoking status according to non-smokers, current smokers, and former smokers after incorporating hidden smokers into the non-smoker group (by cotinine verification) and after incorporating hidden smokers into the former or nonsmoker group (self-reporting smokers).

The STATA software 11.0 (Stata Corp.) was used for statistical analysis, and $\mathrm{P}$ values $<0.05$ were considered to be statistically significant.

\section{Results}

\section{General characteristics of participants}

Of all participants, 4,769 (85.40\%) were non-smokers, $166(2.97 \%)$ were former smokers, $277(4.96 \%)$ were self-reported (current) smokers, and 372 (6.66\%) were cotinine-verified hidden smokers.

The general characteristics of non-smokers, hidden smokers, self-reported (current) smokers, and former smokers are described in Table 1. Median (interquartile range) urine cotinine levels $(\mathrm{mg} / \mathrm{mL})$ of the study participants in the non-smoker, hidden smoker, selfreported (current) smoker, and former smoker groups were 3.56 (0.16-11.42), 124.83 (56.78-63.60), 878.20 (484.60-1450.96), and $3.88(0.49-13.71) \mathrm{ng} / \mathrm{mL}$, respectively. The proportions of patients in each age group were significantly different across smoking groups. There were more women younger than 40 years in the hidden smoker, self-reported (current) smoker, and former smoker groups than in the non-smoker group. The number of patients 65 years and over was relatively small in the hidden smoker group. The proportion of women with an education level below elementary school was $27.7 \%$ among hidden smokers, $40.3 \%$ among nonsmokers, $40.4 \%$ among self-reported (current) smokers, and $46.4 \%$ among former smokers. More women were married among hidden smokers $(78.8 \%)$ and non-smokers $(74.9 \%)$ than among self-reported (current) smokers $(50.9 \%)$ and former smokers $(60.2 \%) .89 .5 \%$ of selfreported (current) smokers had NHI, while $96.8 \%, 93.8 \%$, and $90.4 \%$ of non-smokers, hidden smokers, and nonsmokers, respectively, had NHI. Private health insurance was more common in hidden smokers $(72.6 \%)$ than in the other smoking groups $(\mathrm{p}<0.001)$. Obesity (BMI $\geq 25 \mathrm{~kg}$ / $\mathrm{m}^{2}$ ) and physical activity of moderate intensity were not significantly different between the four groups $(\mathrm{p}=0.106$ and 0.093 , respectively). The proportion of non-drinkers in hidden smoker was smaller than non-smokers and higher than self-reported (current) smokers. The number of who drinks more than 1 drink per month in hidden smokers was relatively more common than non-smokers and less than self-reported (current) smokers. Patients with hypertension and diabetes were more common in the former smoker group than in the other groups, and dyslipidemia was most common in the non-smoker group.

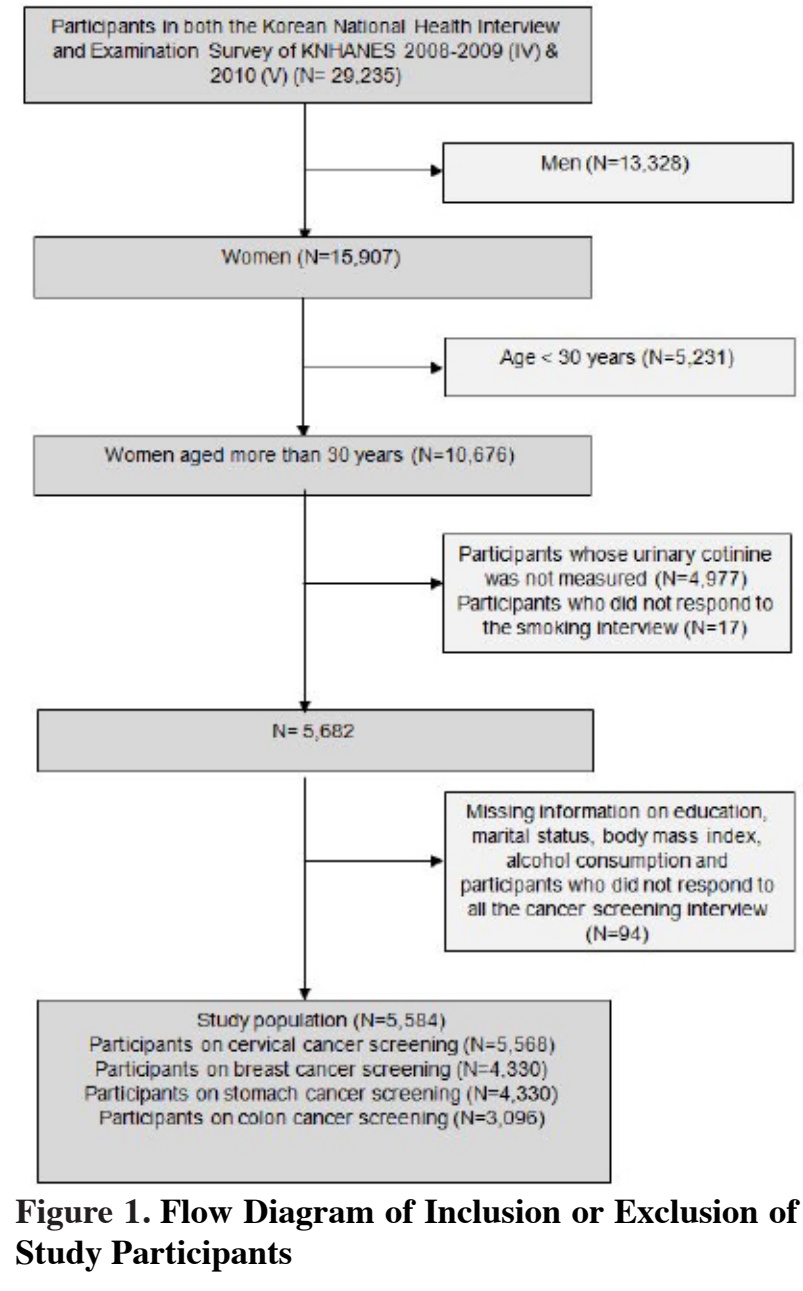

Asian Pacific Journal of Cancer Prevention, Vol 16, 2015 
Young-Jin Ko et al

A diagnosis of cancer was not significantly different between the four groups. The crude cancer-screening rate of cervical, breast, stomach, and colon cancer were $40.7 \%, 39.0 \%, 32.8 \%$, and $14.3 \%$, respectively, in hidden smokers, which were higher than the rates in self-reported (current) smokers and lower than that in non-smokers.

\section{Adherence to cancer screening according to smoking status}

Associations between smoking status and cancer screening in the four groups using multivariate logistic regression are shown in Table 2 . In model 1, we adjusted for socio-demographic factors such as age, BMI, education level, marital status, insurance status, and private insurance. In model 2, we adjusted for socio-demographic factors as well as behavioral risk factors (alcohol consumption and exercise). In model 3, we adjusted for socio-demographic factors, behavioral risk factors, and personal health status (history of chronic disease (e.g. hypertension, diabetes, and dyslipidemia) and history of cancer). Compared to non-smokers, the adjusted odds ratios (aORs) and 95\% confidence intervals (CI) for cervical cancer screening in hidden smokers, self-reported (current) smokers, and former smokers were 0.84 [0.65-1.09], 0.63 [0.47-0.85],

Table 1. General Characteristics of Study Participants According to Smoking Status

\begin{tabular}{|c|c|c|c|c|c|}
\hline & $\begin{array}{l}\text { Non-smoker } \\
(\mathrm{N}=4,769)\end{array}$ & $\begin{array}{l}\text { Hidden smoker* } \\
\qquad(\mathrm{N}=372)\end{array}$ & $\begin{array}{l}\text { Self-reported } \\
\text { (current) smoker } \\
(\mathrm{N}=277)\end{array}$ & $\begin{array}{l}\text { Former smoker } \\
\qquad(\mathrm{N}=166)\end{array}$ & $\mathrm{P}$ value** \\
\hline Urinary cotinine $* * *(\mathrm{ng} / \mathrm{mL})$ & $\begin{array}{r}3.56 \\
(0.16-11.42)\end{array}$ & $\begin{array}{r}124.83 \\
(63.60-556.78)\end{array}$ & $\begin{array}{r}878.2 \\
(484.60-1450.96)\end{array}$ & $\begin{array}{r}3.88 \\
(0.49-13.71)\end{array}$ & $<0.001$ \\
\hline \multicolumn{6}{|l|}{ Age groups } \\
\hline $30-39$ & $986(20.7)$ & $125(33.6)$ & $81(29.2)$ & $62(37.4)$ & \\
\hline $40-49$ & $1,059(22.2)$ & $101(27.2)$ & $58(20.9)$ & $16(9.6)$ & \\
\hline $50-64$ & $1,564(32.8)$ & $80(21.5)$ & $63(22.7)$ & $24(14.5)$ & \\
\hline $65 y e a r s$ and over & $1,160(24.3)$ & $66(17.7)$ & $75(27.1)$ & $24(38.6)$ & $<0.001$ \\
\hline \multicolumn{6}{|l|}{ Education } \\
\hline Less than elementary & $1,923(40.3)$ & $103(27.7)$ & $112(40.4)$ & $77(46.4)$ & \\
\hline Middle/high & $1,965(41.2)$ & $201(54.0)$ & $128(46.2)$ & $58(34.9)$ & \\
\hline College or higher & $881(18.5)$ & $68(18.3)$ & $37(13.4)$ & $31(18.7)$ & 0.014 \\
\hline \multicolumn{6}{|l|}{ Marital status } \\
\hline With spouse & $3,572(74.9)$ & $293(78.8)$ & $141(50.9)$ & $100(60.2)$ & \\
\hline Without spouse & $1,197(25.1)$ & $79(21.2)$ & $136(49.1)$ & $66(39.8)$ & $<0.001$ \\
\hline \multicolumn{6}{|l|}{ National health insurance } \\
\hline NHI & 4,617 (96.8) & $349(93.8)$ & $248(89.5)$ & $150(90.4)$ & \\
\hline Medicaid & $152(3.2)$ & $22(5.9)$ & $29(10.5)$ & $16(9.5)$ & \\
\hline Not involved in either & 0 & $1(0.27)$ & 0 & & $<0.001$ \\
\hline \multicolumn{6}{|l|}{ Private health insurance } \\
\hline No & $3,239(67.9)$ & $270(72.6)$ & $153(55.23)$ & $93(56.0)$ & \\
\hline Yes & $1,470(30.8)$ & $98(26.3)$ & $117(42.2)$ & $72(43.4)$ & \\
\hline "Don’t know" & $60(126)$ & $4(1.1)$ & $7(2.5)$ & $1(0.6)$ & $<0.001$ \\
\hline \multicolumn{6}{|l|}{ Residential area } \\
\hline Urban & $3,433(72.0)$ & $277(74.5)$ & 215 (77.6) & $136(81.9)$ & \\
\hline Rural & $1,336(28.0)$ & $95(25.5)$ & $62(22.4)$ & $30(18.1)$ & $<0.001$ \\
\hline \multicolumn{6}{|l|}{ Obesity } \\
\hline Body mass index $\geq 25 \mathrm{~kg} / \mathrm{m}^{2}$ & $1,520(31.9)$ & $130(35.0)$ & $72(26.0)$ & $64(38.6)$ & 0.106 \\
\hline Body mass index $<25 \mathrm{~kg} / \mathrm{m}^{2}$ & $3,249(68.1)$ & $242(65.1)$ & $205(74.0)$ & $102(61.5)$ & \\
\hline \multicolumn{6}{|l|}{ Physical activity $\dagger$} \\
\hline No & $2,542(53.3)$ & $188(50.5)$ & $166(59.9)$ & $93(56.0)$ & \\
\hline Yes & $2,227(46.7)$ & $184(49.5)$ & $111(40.1)$ & $73(44.0)$ & 0.093 \\
\hline \multicolumn{6}{|l|}{ Alcohol consumption } \\
\hline Non-drinker & $1,239(26.0)$ & $57(15.3)$ & $28(10.1)$ & $10(6.0)$ & \\
\hline Less than 1 drink per month & $1,969(41.3)$ & $141(37.9)$ & $96(34.7)$ & $97(58.4)$ & \\
\hline More than 1 drink per month & $1,561(32.7)$ & $174(46.8)$ & $153(55.2)$ & $59(35.5)$ & $<0.001$ \\
\hline Hypertension $\$$ & $1,147(24.1)$ & $59(15.9)$ & $56(20.2)$ & $55(33.1)$ & $<0.001$ \\
\hline Diabetes $\$$ & $384(8.1)$ & $20(5.4)$ & $14(5.1)$ & $23(13.9)$ & 0.001 \\
\hline Dyslipidemia $†$ & $502(10.5)$ & $21(5.7)$ & $14(5.1)$ & $14(8.4)$ & $<0.001$ \\
\hline History of cancer $\$$ & $193(4.1)$ & $6(1.6)$ & $5(1.8)$ & $7(4.2)$ & 0.079 \\
\hline Cervical cancer screening rate $\S$ & 2119 (44.6) & $151(40.7)$ & $87(31.4)$ & $61(37.0)$ & $<0.001$ \\
\hline Breast cancer screening rate $\S$ & $2,237(46.9)$ & $145(39.0)$ & $79(28.5)$ & $50(30.1)$ & $<0.001$ \\
\hline Stomach cancer screening rate $\S$ & $1,884(39.5)$ & $122(32.8)$ & $73(26.4)$ & $39(23.5)$ & $<0.001$ \\
\hline Colon cancer screening rate $\S$ & $924(19.4)$ & $53(14.3)$ & $37(13.4)$ & $29(17.5)$ & $<0.001$ \\
\hline
\end{tabular}

Data were expressed as number (\%); * Hidden smokers were defined as respondents with urinary cotinine concentrations higher than 50 ng/mL and who reported not to smoke now; ** P values were obtained by $\chi 2$ tests and ANOVA to compare categorical variables and continuous variable, respectively; *** Expressed as median (interquartile range); $†$ Physical activity of moderate intensity is defined by IPAQ short-form questionnaire; † Diagnosed by doctor; $\S$ Stomach cancer: esophagogastroduodenoscopy or Upper GI series within 2 years older than 40 years; Breast cancer: mammography or breast ultrasonography within 2 years older than 40 years; Cervical cancer: pap smear test within 2 years older than 30 years' Colon cancer: Occult blood test within 2 years, Colonoscopy within 10 years, or sigmoidoscopy and double contrast study within 10 years older than 50 years 
and 0.85 [0.59-1.23], respectively, in model 1. After adjusting for socio-demographic factors, behavioral risk factors, and personal health status, the results remained similar. In breast cancer screening, fewer hidden smokers (aOR [95\% CI] $=0.69[0.50-0.95]$ ), self-reported (current) smokers $(\mathrm{aOR}[95 \% \mathrm{CI}]=0.44[0.30-0.64])$, and former smokers $(\mathrm{aOR}[95 \% \mathrm{CI}]=0.60$ [0.37-0.97]) were screened than non-smokers in model 1. After full adjustment, the differences remained significant, with multivariate adjusted ORs [95\%CI] of 0.71 [0.51-0.98], 0.47 [0.320.68 ], and 0.60 [0.37-0.97] respectively, in model 3 . Fewer self-reported (current) smokers underwent stomach cancer screening than did non-smokers (aOR, 0.66; 95\%CI, 0.46-
DOI:http://dx.doi.org/10.7314/APJCP.2015.16.9.4081 Cancer Screening Behavior of Asian Female Hidden Smokers 0.95 in model 3). For colon cancer screening, as compared to non-smokers, the aOR $[95 \% \mathrm{CI}]$ in hidden smokers was 0.58 [0.36-0.93] after adjusting for socio-demographic factors. The marginal significance was observed after full adjustment in model 3 (aOR [95\%CI] $=0.62$ [0.38-1.01]).

Difference in cancer screening adherence by classification of hidden smokers into the "current smoker" group or the "former or non-smoker" group

The associations between smoking status and cancer screening using multivariate logistic regression after incorporating hidden smokers into the current smoker (by cotinine verification) group and after incorporating hidden

Table 2. Association between Smoking Status and Cancer Screening using Multivariate Logistic Regression

\begin{tabular}{|c|c|c|c|c|c|}
\hline Smoking status & $\mathrm{N}$ & $\begin{array}{c}\text { weighted proportion } \\
(\%(\mathrm{SE}))\end{array}$ & $\begin{array}{c}\text { Model 1** } \\
\text { aOR }(95 \% \mathrm{CI})\end{array}$ & $\begin{array}{c}\text { Model 2*** } \\
\text { aOR }(95 \% \mathrm{CI})\end{array}$ & $\begin{array}{c}\text { Model } 3 \dagger \\
\operatorname{aOR}(95 \% \mathrm{CI})\end{array}$ \\
\hline \multicolumn{6}{|l|}{ Cervical cancer screening } \\
\hline Non-smoker & 4,755 & $46.0(0.8)$ & 1 & 1 & 1 \\
\hline Hidden smoker * & 371 & $43.9(3.0)$ & $0.84(0.65,1.09)$ & $0.84(0.65,1.09)$ & $0.85(0.66,1.10)$ \\
\hline Self-reported (current) smoker & 277 & $32.6(3.2)$ & $0.63(0.47,0.85)$ & $0.64(0.46,0.86)$ & $0.64(0.47,0.87)$ \\
\hline Former smoker & 165 & $40.5(4.5)$ & $0.85(0.59,1.23)$ & $0.86(0.59,1.24)$ & $0.86(0.60,1.25)$ \\
\hline \multicolumn{6}{|l|}{ Breast cancer screening } \\
\hline Non-smoker & 3,783 & $53.4(1.0)$ & 1 & 1 & 1 \\
\hline Hidden smoker $*$ & 247 & $46.6(3.8)$ & $0.69(0.50,0.95)$ & $0.70(0.51,0.97)$ & $0.71(0.51,0.98)$ \\
\hline Self-reported (current) smoker & 196 & $31.8(3.8)$ & $0.44(0.30,0.64)$ & $0.46(0.32,0.66)$ & $0.47(0.32,0.68)$ \\
\hline Former smoker & 104 & $34.9(5.5)$ & $0.60(0.37,0.97)$ & $0.60(0.37,0.97)$ & $0.60(0.37,0.97)$ \\
\hline \multicolumn{6}{|l|}{ Stomach cancer screening } \\
\hline Non-smoker & 3,783 & $45.0(1.0)$ & 1 & 1 & 1 \\
\hline Hidden smoker $*$ & 247 & $38.9(3.7)$ & $0.73(0.53,1.01)$ & $0.74(0.54,1.02)$ & $0.75(0.54,1.03)$ \\
\hline Self-reported (current) smoker & 196 & $31.6(3.8)$ & $0.63(0.44,0.90)$ & $0.65(0.45,0.94)$ & $0.66(0.46,0.95)$ \\
\hline Former smoker & 104 & $34.0(5.6)$ & $0.78(0.48,1.26)$ & $0.77(0.47,1.24)$ & $0.77(0.47,1.24)$ \\
\hline \multicolumn{6}{|l|}{ Colon cancer screening } \\
\hline Non-smoker & 2,724 & $33.2(1.1)$ & 1 & 1 & 1 \\
\hline Hidden smoker * & 146 & $33.7(4.6)$ & $1.02(0.67,1.56)$ & $1.05(0.69,1.61)$ & $1.07(0.71,1.67)$ \\
\hline Self-reported (current) smoker & 138 & $20.6(3.8)$ & $0.58(0.36,0.93)$ & $0.59(0.37,0.98)$ & $0.62(0.38,1.01)$ \\
\hline Former smoker & 88 & $26.5(5.2)$ & $1.00(0.58,1.71)$ & $1.01(0.58,1.74)$ & $1.01(0.59,1.75)$ \\
\hline
\end{tabular}

All data are weighted to the residential population of Korea; * Hidden smokers were defined as respondents with urinary cotinine concentrations higher than $50 \mathrm{ng} / \mathrm{mL}$ and who reported not to smoke now; ** Model 1 adjusted for age, body mass index, education level, marital status, insurance status, and private insurance; *** Model 2 adjusted for age, body mass index, education level, marital status, insurance status, private insurance, alcohol drinking, and exercise; $\dagger$ Model 3 adjusted for age, body mass index, education level, marital status, insurance status, private insurance, alcohol drinking, exercise, history of chronic disease (hypertension, diabetes, and dyslipidemia), and history of cancer

Table 3. Difference in Cancer Screening Adherence by Classification of Hidden Smokers into "Current Smoker" (by Cotinine-Verification) or "Former Or Non-Smoker" Groups (Classified Same as Self-Report)

\begin{tabular}{|c|c|c|c|c|c|c|c|}
\hline & & \multicolumn{3}{|c|}{$\begin{array}{l}\text { After incorporating hidden smoker } \\
\text { into current smoker }\end{array}$} & \multicolumn{3}{|c|}{$\begin{array}{l}\text { After incorporating hidden smoker } \\
\text { into former or non-smoker }\end{array}$} \\
\hline & & $\mathrm{N}$ & Weighte & $\mathrm{aOR}(95 \% \mathrm{CI})$ & $\mathrm{N}$ & Weighted & $\mathrm{aOR}(95 \% \mathrm{CI})$ \\
\hline & & & nertion 0 & & & & \\
\hline \multirow[t]{3}{*}{ Cervical cancer screening } & Non-smoker & 4755 & $46.0(0.8)$ & 1 & 5126 & $45.9(0.8)$ & 1 \\
\hline & Current smoker & 648 & $39.0(2.2)$ & $0.76(0.62,0.93)$ & 277 & $32.6(3.2)$ & $0.65(0.48,0.89)$ \\
\hline & Former smoker & 165 & $40.5(4.5)$ & $0.86(0.59,1.25)$ & 165 & $40.5(4.5)$ & $0.88(0.61,1.27)$ \\
\hline \multirow[t]{3}{*}{ Breast cancer screening } & Non-smoker & 3783 & $53.4(1.0)$ & 1 & 4030 & $53.0(0.9)$ & 1 \\
\hline & Current smoker & 443 & $40.1(2.7)$ & $0.59(0.47,0.76)$ & 196 & $31.8(3.8)$ & $0.48(0.33,0.70)$ \\
\hline & Former smoker & 104 & $34.9(5.5)$ & $0.60(0.37,0.98)$ & 104 & $34.9(5.5)$ & $0.61(0.38,0.99)$ \\
\hline \multirow[t]{3}{*}{ Stomach cancer screening } & Non-smoker & 3783 & $45.0(1.0)$ & 1 & 4030 & $44.6(0.9)$ & 1 \\
\hline & Current smoker & 443 & $35.8(2.7)$ & $0.71(0.56,0.91)$ & 196 & 31.6 & $0.67(0.47,0.97)$ \\
\hline & Former smoker & 104 & $34.0(5.6)$ & $0.77(0.47,1.25)$ & 104 & $34.0(5.6)$ & $0.78(0.48,1.27)$ \\
\hline \multirow[t]{3}{*}{ Colon cancer screening } & Non-smoker & 2724 & $33.2(1.1)$ & 1 & 2870 & $33.2(1.0)$ & 1 \\
\hline & Current smoker & 284 & $27.1(3.0)$ & $0.85(0.61,1.17)$ & 138 & $20.6(3.8)$ & $0.62(0.38,1.01)$ \\
\hline & Former smoker & 88 & $26.5(5.2)$ & $1.02(0.59,1.76)$ & 88 & $26.5(5.2)$ & $1.01(0.59,1.74)$ \\
\hline
\end{tabular}

All data are weighted to the residential population of Korea; ORs were calculated using multivariate logistic regression adjusted for age, body mass index, education level, marital status, insurance status, private insurance, alcohol drinking, exercise, history of chronic disease (hypertension, diabetes, and dyslipidemia), and history of cancer 
smokers into the former or non-smoker (classified same as self-report) groups after adjusting for age, BMI, education level, marital status, insurance status, private insurance, alcohol drinking, exercise, history of chronic disease (hypertension, diabetes, and dyslipidemia), and history of cancer are shown in Table 3 . When hidden smokers were included in the current smoker group, multivariate adjusted ORs [95\% CI] of self-reported (current) smokers were 0.76 [0.62-0.93], 0.59 [0.47-0.76], 0.71 [0.56-0.91], and 0.85 [0.61-1.17] for cervical cancer, breast cancer, stomach cancer, and colon cancer screening, respectively, compared to non-smokers. When hidden smokers were included in the former smoker or non-smoker groups, the significance became prominent (aORs [95\%CI] of self-reported (current) smoker versus non-smoker: 0.65 [0.48-0.89], 0.48 [0.33-0.70], 0.67 [0.47-0.97], and 0.62 [0.38-1.01] for cervical cancer, breast cancer, stomach cancer, and colon cancer screening, respectively).

\section{Discussion}

In the present study, $372(6.7 \%)$ of 5,584 women answered that they did not currently smoke on the questionnaire, but were classified as hidden smokers based on cotinine verification. Self-reported (current) female smokers showed significantly less compliance to screening guidelines than non-smokers for cervical, breast, and stomach cancer screening $(\mathrm{aORs}[95 \% \mathrm{CI})=0.64$ [0.47-0.87], 0.47 [0.32-0.68], and 0.66 [0.46-0.95], respectively), and marginally significantly less compliance for colon cancer screening $(\mathrm{aOR}[95 \% \mathrm{CI}]=0.62[0.38$ 1.01]). The data indicates that hidden smokers have lower adherence to breast, cervical, and stomach cancer screening. Although statistical significance was observed only for breast cancer screening $(\mathrm{aOR}[95 \% \mathrm{CI}]=0.71$ [0.51-0.98]), the stomach and cervical cancer screening rates were also marginally lower in hidden smokers than in non-smokers after adjusting for major confounding factors. Moreover, the lower adherence to cancer screening of current smokers was attenuated after we incorporated hidden smokers (formerly included in the non-smoker group) into current smokers.

A recent study in Korea showed that hidden smokers tend to be younger, live with their parents or a spouse rather than alone or with others, and have higher education and non-manual occupations, which was consistent with the findings of our study (Jung-Choi et al., 2012). Additionally, our findings are similar to those of previous studies showing an association between smoking status and cancer screening. Smoking is related to unhealthy behavior. Current smokers tend to have less favorable health behavior such as lower physical activity, unhealthy diet, and alcohol abuse (Larkin et al., 1990; Schumann et al., 2001; Berrigan et al., 2003; de Vries et al., 2008). Smoking status has also been shown to be significantly associated with breast cancer screening in Korea (Lee et al., 2010). Lee et al showed that the adherence rate to breast cancer screening was $31.98 \%$ in lifetime non-smokers and $14.89 \%$ in lifetime smokers, with a multivariate adjusted OR [95\%CI] for smokers of 0.52 [0.35-0.79] compared to non-smokers using KNHANES
III data (Lee et al., 2010). Many studies have reported differences in psychosocial variables between smokers and non-smokers, which seem to influence health-behavior decisions (Marteau et al., 2002; McKee, 2009). Myong et al showed that approximately $30.0 \%$ and $33.8 \%$ of smokers and nonsmokers, respectively, adhere to colorectal cancer screening, based on KNHANES IV data for both men and women (Myong et al., 2012). The lack of difference seen in adherence to colorectal screening may be due to different definitions of screening criteria and inclusion of both sexes.

In addition, defining smokers based on self-reporting could be inaccurate due to cultural considerations in Asian populations. Although population-based surveys using self-reporting to identify smokers are generally regarded as reliable in Western countries (West et al., 2007; Yeager and Krosnick, 2010; Wong et al., 2012), they significantly underestimate the number of smokers in Asian countries (Wang and Ma, 2012). In a survey in Shanghai, China, Wang et al reported that self-reporting captured only $65 \%$ of smokers among adult women and only $37 \%$ among female students using the capture-recapture method among 11,104 students (aged 12-20 years) and their parents (aged $\geq 35$ years) (Wang and Ma, 2012). Ma et al (Ma et al., 2013) also showed that the prevalence of smoking among girls was $5.2 \%$ by self-reported smokers and $14.2 \%$ by the capture-recapture method and concluded that self-reporting to identify smokers among Chinese adolescents may significantly underestimate the number of smokers. In a study including 322 Korean university students, a significant difference was found between the results reported by the questionnaire and data from urine tests (Lee et al., 2009). Recently, Jung-Choi et al showed that self-reported female smokers made up less than half of cotinine-verified smokers and suggested that biochemical verification needs to be considered with national tobacco surveys in Asian countries (JungChoi et al., 2012). Therefore, we defined smokers using biochemical verification methods. In the present study, hidden (cotinine-verified) smokers were defined as respondents with urinary cotinine concentrations higher than $50 \mathrm{ng} / \mathrm{mL}$ and who reported to not smoke. This underreporting may also be affected by cultural backgrounds in which smoking is stigmatized among certain subgroups (Jung-Choi et al., 2012). For example, the influence of Confucianism, resulting in social repression of women's smoking, is considered a major reason for the low rate of tobacco use among Korean women (Cho et al., 2008).

We also analyzed the cancer screening behavior of subjects in smoking groups (non-smokers, current smokers, and former smokers) defined only by selfreported questionnaire. When we incorporated hidden smokers into the non-smoker group, the current smoker group showed even less compliance to screening guidelines than non-smokers. However, this significant association was slightly attenuated when hidden smokers were incorporated into the current smoker group. In fact, reports on female smokers defined by self-reporting misclassify subjects into smoking status groups. Therefore, cotinine verification is important to accurately define smoking status among Asian women status. 
The present study had several limitations. First, the study was conducted using a cross-sectional design and therefore causal relationships could not be determined. Second, the number of cotinine-verified hidden female smokers was too small to investigate exact associations, which explains the marginal significance of low cervical and stomach cancer screening in hidden smokers. However, our study also has several strengths. First, we derived data from the KNHANES, which area series of national health survey in Korea. Second, we used a cotinine-verified method to identify female hidden smoker.

This study suggests that hidden smokers should be identified and endeavors taken to improve adherence to cancer screening in hidden smokers in order to increase cancer screening compliance. Perception of cancer risk and internal motivation to emphasize the individual's willingness were reported to improve screening adherence (Jung and Jo, 2014; Kim et al., 2014). To maintain cancer screening continuously, education to strengthen internal motivation factors and to perceive cancer risk among hidden smokers should be needed. Moreover, former smokers showed less screening adherence than nonsmokers but more than current smokers. This suggests that policies to improve cancer-screening adherence should be targeted not only to improve adherence of female smokers but also to convert smokers into former smokers.

\section{References}

Berrigan D, Dodd K, Troiano RP, et al (2003). Patterns of health behavior in U.S. adults. Prev Med, 36, 615-23.

Botteri E, Iodice S, Bagnardi V, et al (2008). Smoking and colorectal cancer: a meta-analysis. JAMA, 300, 2765-78.

Carlos RC, Fendrick AM, Patterson SK, et al (2005). Associations in breast and colon cancer screening behavior in women. Acad Radiol, 12, 451-8.

Centers for Disease C, Prevention (2008). Smoking-attributable mortality, years of potential life lost, and productivity losses--United States, 2000-2004. Morb Mortal Wkly Rep, 57, 1226-8.

Cho HJ, Khang YH, Jun HJ, et al (2008). Marital status and smoking in Korea: the influence of gender and age. Soc Sci Med, 66, 609-19.

de Vries H, van 't Riet J, Spigt M, et al (2008). Clusters of lifestyle behaviors: results from the Dutch SMILE study. Prev Med, 46, 203-8.

Gaudet MM, Gapstur SM, Sun J, et al (2013). Active smoking and breast cancer risk: original cohort data and metaanalysis. J Natl Cancer Inst, 105, 515-25.

Haufroid V, Lison D (1998). Urinary cotinine as a tobacco-smoke exposure index: a minireview. Int Arch Occup Environ Health, 71, 162-8.

International Collaboration of Epidemiological Studies of Cervical Cancer (2007). Comparison of risk factors for invasive squamous cell carcinoma and adenocarcinoma of the cervix: collaborative reanalysis of individual data on 8,097 women with squamous cell carcinoma and 1,374 women with adenocarcinoma from 12 epidemiological studies. Int J Cancer, 120, 885-91.

International Collaboration of Epidemiological Studies of Cervical Cancer, Appleby P, Beral V, et al (2006). Carcinoma of the cervix and tobacco smoking: collaborative reanalysis of individual data on 13,541 women with carcinoma of the cervix and 23,017 women without carcinoma of the cervix
DOI:http://dx.doi.org/10.7314/APJCP.2015.16.9.4081

Cancer Screening Behavior of Asian Female Hidden Smokers

from 23 epidemiological studies. Int J Cancer, 118, 1481-95.

Johnson KC, Miller AB, Collishaw NE, et al (2011). Active smoking and secondhand smoke increase breast cancer risk: the report of the Canadian Expert Panel on Tobacco Smoke and Breast Cancer Risk (2009). Tob Control, 20, 2.

Jun-Sik N (2010). National Cancer Screening Program. J Korean Medical Association, 53, 386-91.

Jung-Choi KH, Khang YH, Cho HJ (2012). Hidden female smokers in Asia: a comparison of self-reported with cotinineverified smoking prevalence rates in representative national data from an Asian population. Tob Control, 21, 536-42.

Jung SM, Jo HS (2014). Intrinsic motivation factors based on the self-determinant theory for regular breast cancer screening. Asian Pac J Cancer Prev, 15, 10101-6.

Kim JH, Park EC, Yoo KB (2014). Impact of perceived cancer risk on the cancer screening rate in the general korean population: results from the korean health panel survey data. Asian Pac J Cancer Prev, 15, 10525-9.

Korea Centers for Disease C, Prevention, (2007). Guide to the utilization of the data from the third Korea National Health and Nutrition Examination Survey.

Korea Centers for Disease C, Prevention, (2010). Korean National Health and Nutrition Examination Survey. 2010 http://knhanes.cdc.go.kr/include/IFrameNull.jsp?url 1/4 / board/Download/

Ladeiras-Lopes R, Pereira AK, Nogueira A, et al (2008). Smoking and gastric cancer: systematic review and metaanalysis of cohort studies. Cancer Causes Control, 19, 689-701.

Larkin FA, Basiotis PP, Riddick HA, et al (1990). Dietary patterns of women smokers and non-smokers. J Am Diet Assoc, 90, 230-7.

Lee CY, Shin S, Lee HK, et al (2009). Validation of self-report on smoking among university students in Korea. Am J Health Behav, 33, 540-9.

Lee K, Lim HT, Park SM (2010). Factors associated with use of breast cancer screening services by women aged >or= 40 years in Korea: the third Korea National Health and Nutrition Examination Survey 2005 (KNHANES III). BMC Cancer, 10, 144

Ma J, Zhu J, Li N, et al (2013). Severe and Differential Underestimation of Self-reported Smoking Prevalence in Chinese Adolescents. Int J Behav Med, 21, 662-6.

Marteau TM, Hankins M, Collins B (2002). Perceptions of risk of cervical cancer and attitudes towards cervical screening: a comparison of smokers and non-smokers. Fam Pract, 19, 18-22.

McKee SA (2009). Developing human laboratory models of smoking lapse behavior for medication screening. Addict Biol, 14, 99-107.

Myong JP, Shin JY, Kim SJ (2012). Factors associated with participation in colorectal cancer screening in Korea: the Fourth Korean National Health and Nutrition Examination Survey (KNHANES IV). Int J Colorectal Dis, 27, 1061-9.

Rakowski W, Clark MA, Truchil R, et al (2005). Smoking status and mammography among women aged 50-75 in the 2002 behavioral risk factor surveillance system. Women Health, 41, 1-21.

Rakowski W, Meissner H, Vernon SW, et al (2006). Correlates of repeat and recent mammography for women ages 45 to 75 in the 2002 to 2003 health information national trends survey (HINTS 2003). Cancer Epidemiol Biomarkers Prev, 15, 2093-101.

Ruidavets JB, Bataille V, Dallongeville J, et al (2004). Alcohol intake and diet in France, the prominent role of lifestyle. Eur Heart J, 25, 1153-62.

Schumann A, Hapke U, Rumpf HJ, et al (2001). The association 
between degree of nicotine dependence and other health behaviours. Findings from a German general population study. Eur J Public Health, 11, 450-2.

Shapiro JA, Seeff LC, Nadel MR (2001). Colorectal cancerscreening tests and associated health behaviors. Am J Prev Med, 21, 132-7.

SNRT Subcommittee on Biochemical Verification (2002). Biochemical verification of tobacco use and cessation. Nicotine Tob Res, 4, 149-59.

The IPAQ Group (2005). Guidelines for data processing and analysis of the international physical activity questionnaire (IPAQ) - Short and Long Forms

Vineis P, Alavanja M, Buffler P, et al (2004). Tobacco and cancer: recent epidemiological evidence. J Natl Cancer Inst, 96, 99-106.

Wang Z, Ma J (2012). Prevalence and patterns of tobacco use in Asia. Lancet, 380, 1905-6.

West R, Zatonski W, Przewozniak K, et al (2007). Can we trust national smoking prevalence figures? Discrepancies between biochemically assessed and self-reported smoking rates in three countries. Cancer Epidemiol Biomarkers Prev, 16, 820-2.

World Health Organization (2000). World health organization regional office for the western pacific region, international association for the study of obesity, international obesity task force. the asian-pacific perspective: redefining obesity and its treatment. sydney: health communications Australia.

World Health Organization (2011). Who report on the global tobacco epidemic, 2011: Warning about the dangers of tobacco. 2013.06.14.

Wong SL, Shields M, Leatherdale S, et al (2012). Assessment of validity of self-reported smoking status. Health Rep, 23, 47-53.

Yeager DS, Krosnick JA (2010). The validity of self-reported nicotine product use in the 2001-2008 national health and nutrition examination survey. Med Care, 48, 1128-32. 